\title{
Pronouncing Text by Analogy
}

\author{
Robert I. Damper and John F.G. Eastmond \\ Image, Speech and Intelligent Systems (ISIS) Research Group, \\ Department of Electronics and Computer Science, \\ University of Southampton, \\ Southampton SO17 1BJ, \\ UK. \\ \{ridlje\}@ecs.soton.ac.uk
}

\begin{abstract}
Pronunciation-by-analogy $(\mathrm{PbA})$ is an emerging technique for text-phoneme conversion based on a psychological model of reading aloud. This paper explores the impact of certain basic implementational choices on the performance of various $\mathrm{PbA}$ models. These have been tested on their ability to pronounce sets of short pseudowords previously used in similar studies, as well as lexical words temporarily removed from the dictionary. Best results of $85.7 \%$ and $67.9 \%$ words correct are obtained for the pscudowords and lexical words respectively, casting doubt on certain previous-reported performance figures in the literature.
\end{abstract}

\section{Introduction}

Pronunciation-by-analogy $(\mathrm{PbA})$ is an influential psychological model of the process of reading aloud. In $\mathrm{PbA}$, most words are pronounced by retricving their phonemic form from the readers's lexicon, or dictionary. The pronunciation for a 'novel' word not in the lexicon, however, is derived not by the application of abstract letter-to-sound rules but is 'assembled' from the (known) pronunciations of words that it resembles. $\mathrm{PbA}$ has obvious application to text-to-speech conversion by machine.

Although $\mathrm{PbA}$ programs have been presented in the literature, they are they are few in number. Dedina and Nusbaum (1991) describe PRONOUNCE: a rather simple system for English. Sullivan and Damper $(1990 ; 1992 ; 1993)$ describe a considerably more complex and developed system, but which apparently yields a much poorer performance.

As a psychological theory, $\mathrm{PbA}$ is under-specified: offering little meaningful guidance on the implementation choices which confront the programmer. Indeed, Sullivan and Damper (1993) show that such choices can have a profound impact on performance. In this paper, we seek to understand how Dedina and Nusbaum's largely unjustified implementational choices affected their results and, thereby, to resolve the conflict between their performance claims and Sullivan and Damper's.

\section{Psychological Background}

In the standard dual-route model of reading aloud (Coltheart, 1978), there is a lexical route for the pronunciation of known words and a parallel route utilising abstract letter-to-sound rules for the pronunciation of unknown ('novel') words. Arguments for dual-route theory cite the ability to pronounce pseudowords (non-words conforming to the spelling patterns of English), latency difference effects between regular and exception words, and apparent double dissociation between the two routes in dyslexia (see Humphreys and Evett, 1985). However, all these observations can arguably be explained by a single route. One pervasive idea is that pseudowords are pronounced by analogy with lexical words that they resemble (Baron, 1977; Brooks, 1977; Glushko, 1979; 1981; Brown and Besner, 1987). Glushko, for instance, showed that "exception pseudowords" like tave take longer to read than "regular pseudowords" such as taze. Here, taze is considered as a "regular pseudoword" since all its orthographic 'neighbours' (raze, gaze, maze etc.) have the regular vowel pronunciation /el/. By contrast, tave is considered to be an "exception pseudoword" since it has the exception word (have, /hav/) as an orthographic neighbour. Thus, according to Glushko (1979), the "assignment of phonology to non-words is open to lexical influence". This is at variance with the notion of two independent routes to pronunciation. Instead:

\footnotetext{
"it appears that words and pscudowords are pronounced using similar kinds of orthographic and phonological knowledge: the pronunciation of words that share orthographic features with them, and specific spelling-to-sound rules for multiletter spelling patterns."
} 
There are two forms of PbA: explicit analogy (Baron, 1977) is a conscious strategy of recalling a similar word and moditying its pronunciation, whereas in implicit analogy (Brooks, 1977) a pronunciation is derived from generalised phonographic knowledge about existing words. The latter has obvious commonalitics with most single-route, comnectionist models (e.g. Sejnowski and Rosenberg, 1987) in which the generalised knowledge is learned (c.g. by backpropagation) as a set of weights, and the network has no holistic notion of the concept 'word'.

Until the recent advent of computational $\mathrm{Pb} \triangle \mathrm{mod}$ cls, analogy 'theory' could only be considered seriously underspecified. Cleatly, its operation must depend critically on some measure of similarity, and "without a metric for similarity and without a specification of how similar is similar enough, the concept of analogy by similarity offers little insight" (Glushko, 1981, p. 72). liurther, as detailed by Brown and Besner (1987), the operation of lexical analogy must be constrained by factors such as:

- the size of the segment shared between novel and lexical word;

- its position in the two strings;

- its frequency of occurrence in the language;

- and the frequency of occurrence of the words containing it;

none of which had then received serious consideration. Accordingly, they write: "Extant analogy models are not capable of predicting the outcome of" assembly op)erations for all possible strings."

In particular, the 'theory' gives no principled way of deciding the orthographic neighbours of a novel word which are decmed to influence its pronunciation whereas a computational model must (specifically or otherwise) do so.

\section{Existing l'bA Programs}

\subsection{Dedina and Nusbaum's System}

The overall structure of PRONOUNCH is as shown in Fig. 1. The Iexical database consists of "approximately 20,000 words based on Webster's Pocket Dictionary" in which text and phonemes have been automatically aligned. Dedina and Nusbaum acknowledge the crude nature of their alignment procedure, saying it "was carried out by a simple Lisp program that only uses knowledge about which phonemes are consonants and which are vowels."

An input string is matched in turn against all orthographic entries in the lexicon. The process starts with the input string and the current dictionary entry leftaligned. Information about matching letter substrings

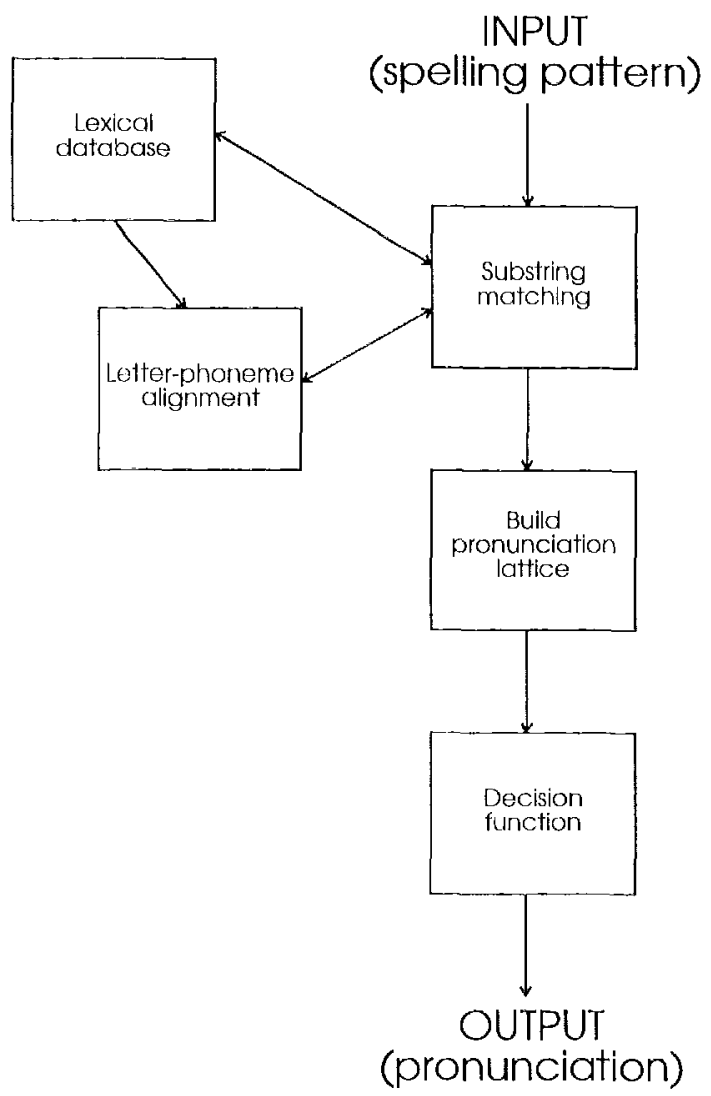

lïgure 1: Dedina and Nusbaum's PRONOUNCE.

- and their corresponding phoneme substrings in the dictionary entry under consideration - is entered into a pronunciation lattice as detailed below. The shorter of the two strings is then shifted right by one letter and the process repeated. This continues until the two are right-aligned, i.c. the number of right shifts is equal to the difference in length between the two strings. The process is repeated for all words in the dictionary.

A node of the lattice represents a matched letter, $L_{i}$, at some position, $i$, in the input, as illustrated in Fig. 2. The node is labelled with its position index $i$ and with the phoneme which corresponds to $L_{i}$ in the malched substring, $l_{i m}$ say, for the $m$ th matched substring. An are is placed from node $i$ to node $j$ if there is a matched substring starting with $L_{i}$ and ending with $L_{j}$. 'The are is labelled with the phonemes intermediate between $P_{i m}$ and $P_{j m}$ in the phoneme part of the matched substring. Note that the empty string labels ares corresponding to bigrams: the two symbols of the bigram label the nodes at either end. Additionally, arcs are labelled with a "frequency" count which is incremented by one each time that substring (with that pronunciation) is matched during the pass through the lexicon. Finally, there is a Start node at position () and an End node at position one greater than the length of the input string. 


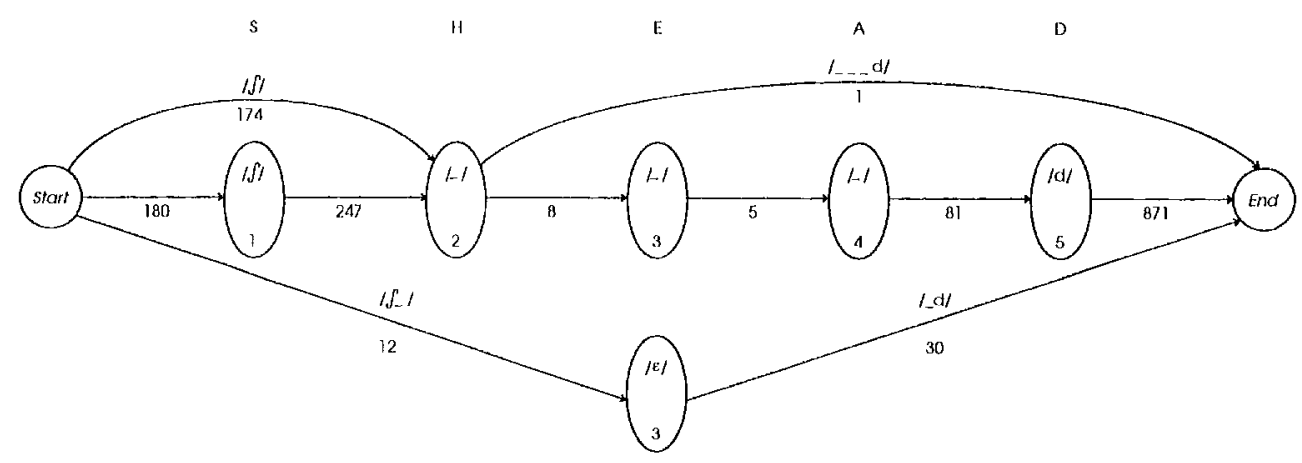

Figure 2: Partial pronunciation lattice for the pseudoword shead.

A possible pronunciation for the input corresponds to a complete path through its lattice from Start to End, with the output string assembled by concatenating in order the phoneme labels on the nodes/arcs. The set of candidate pronunciations is then passed to the decision function. Two (prioritised) heuristics are used to rank the pronunciations, and the top-ranking candidate selected as the output. The first is based on path length. If one candidate corresponds to a unique shortest path (in terms of number of arcs) through the lattice, this is selected as the output. Otherwise, candidates that tie are ranked on the sum of their arc "frequencies".

Dedina and Nusbaum tested PRONOUNCE on 70 of Glushko's (1979) pseudowords, which "were four or five characters long and were derived from monosyllabic words by changing one letter". Seven subjects with phonetics training were asked to read these and give a transcription for the first pronunciation which came to mind. A 'correct' pronunciation for a given pseudoword was considered to be one produced by any of the subjects. A word error rate of $9 \%$ is reported.

\subsection{Sullivan and Damper's System}

Sullivan and Damper employ a more principled alignment procedure based on the Lawrence and Kaye (1986) algorithm. By pre-computing mappings and their statistics, they implemented a considerably more 'implicit' form of $\mathrm{PbA}$ : there is no explicit matching of the input string with lexical entries. Their pronunciation lattice differs, with nodes representing junctures between symbols and arcs representing letterphoneme mappings. They also examine different ways of numerically ranking candidates, taking into account probabilities estimates for the letter-phoneme mappings used in the assembled pronunciation.

Given the improved alignment and candidateranking methods, better performance than Dedina and Nusbaum might be expected. On the contrary, Sullivan and Damper's best result on the full set of 131 pseudowords from Glushko (1979) (plus another 5 words -see section 5.1$)$ is only $70.6 \%(1993$, p. 449$)$. This is an error rate of almost $30 \%$, as compared to Dedina and Nusbaum's $9 \%$ on the smaller test set of size 70. Diflerences in test-set size and between British and American English, the transcription standards of the phoneticians, and the lexicons employed seem insufficient to explain this.

\section{Re-Implementing PRONOUNCE}

Our purpose was to re-implement PRONOUNCE, assess its performance, and study the impact of various implementational choices on this performance. However, the described alignment algorithm is problematic (see pp. 71-73 of Sullivan, 1992) and needs to be replaced. Rather than re-implement a flawed algorithm, we have used manually-aligned data. Since manual alignment generally produces a better result than automatic alignment, we ought to produce an even lower error rate than Dedina and Nusbaum's claimed $9 \%$.

The performance on lexical words (temporarily removed from the lexicon) has not previously been assessed but seems worthwhile. Arguably, 'real' words form a much more sensible test set for a PbA system than pseudowords, not least because they are multisyllabic. Temporary removal from the lexicon means that the pronunciation must be assembled by theianalogy process rather that merely retrieved in its entirety. Hence, we believe it is sensible and important to test any $\mathrm{PbA}$ system in this way.

\subsection{Lexical Databases}

To examine any impact that the specific lexical database might have on performance, we have used two in this work: the 20,009 words of Webster's Pocket Dictionary and the 16,280 words of the Teacher's Word Book (TWB) (Thorndike and Lorge, 1944). In both cases, letters and phonemes have previously been hand-aligned for the purposes of training backpropagation networks. The Webster's database is that used by Sejnowski and Rosenberg (1987) to train and test NETtalk. The TWB database is that used by McCulloch, Bedworth and Bridle (1987) for NETspeak. 
The phoneme inventory is of size 52 in both cases, including the null phoneme but excluding stress symbols. We leave the very important problem of stress assignment for later study.

\subsection{Re-Implementation Details}

The re-implementation was programmed in $\mathrm{C}$ on a Hewlett-Packard 712/80 workstation running HP-UX. A 'direct' version scores candidates using Dedina and Nusbaum's method with its two prioritised heuristics: we call this model D\&N. Two other methods for scoring have also been implemented. In one, we replace the sccond (maximum sum) heuristic with the maximum product of the are frequencies: we call this model PROD. (It still selects primarily on the basis of shortest path length.) We have also implemented a version which uses a single heuristic. This takes the product along each possible path from Start to End of the mapping probabilities for that arc. These are computed using Method 1 (a priori version) of Sullivan and Damper (1993, pp. 446-447). For all paths corresponding to the same pronunciation, these values are summed to give an overall score for that pronunciation. We call this the MP model. The final product score is not a proper probability for the assembled pronunciation, since the scores do not sum to one over all the candidates.

The 'best' pronunciation is found by depth-first search of the lattice, implemented as a preorder tree traversal. For the D\&N and PROD models, paths were pruned when their length exceeded the shortest found so far for that input, leading to a useful reduction in run times. A similarly motivated pruning was carried out for the MP model. If any product fell below a threshold during traversal, its corresponding path was discarded. The threshold used was $\epsilon$ times the maximum product score found so far, with $\epsilon$ set by at $10^{-3}$. While this may have led to the pruning of a path contributing to the 'best' pronunciation, its contribution would be very small. Again, this gave a very significant improvement in run times for the testing of lexical words (section 5.2 below) but was unnecessary for the testing of pseudowords.

\section{Results}

\subsection{Pseudowords}

Pronunciations have been obtained for:

- the 70 pseudowords from Glushko (1979) used by Dedina and Nusbaum to test PRONOUNCE. The 'correct' pronunciation for these strings is taken to be that given by Dedina and Nusbaum (1991, pp. 61-62). We refer to this test set as D\&N 70 .

- the full set of 131 pseudowords from Glushko plus two others (goot, pome) plus two lexical words (cat and play) plus the pseudohomophone $k w i k$, as used by Sullivan (1992). The 'correct' pronunciations are those read aloud by Sullivan's 20 non-phonetician subjects, and transcribed by him as British Received Pronunciation. We refer to this test set as Sull 136. Our expectation is that the crror rate will be relatively high for this test set, partly because of its larger size but more importantly because the subjects' dialect of English is British RP rather that general American, i.c. there is a very significant inconsistency with the lexical databases.

The output has been scored on words correct and also on symbol score (i.e. phonemes correct) using the Levenshtein (1966) string-edit distance as shown in Table 1.

Our best comparison with Dedina and Nusbaum (D\&N 70 test set, D\&N model, Webster's database) grives a figure of $77.1 \%$ words correct. This is enormously poorer than their approximately $91 \%$ words correct - yet the implementation, reference pronunciations and test set are (as far as we can tell) identical. The only relevant difference is that the Webster's database is automatically-aligned in their work and handaligned in ours. The clear expectation, given the crude nature of their alignment, is that they should have experienced a higher crror rate, not a dramatically lower one. Overall, this result accords far more closely with Sullivan and Damper (1993) whose best word score for automatic alignment (and using smaller databases but a larger test set) was just over $70 \%$.

The re-implementation made 16 errors under the above conditions. Dedina and Nusbaum's claim of $9 \%$ words correct amounts to just 6 errors, 3 of which are the same as ours. The commonest problem is vowel substitution. It is possible to discount a very few errors as essentially trivial, reducing the error rate marginally to some $20 \%$. We conclude, therefore, that Dedina and Nusbaum's reported crror rate of $9 \%$ is unattainable.

In our opinion, a major deficiency of the simple shortest-path length heuristic is that the output can become unreasonably sensitive to rare or unique pronunciations. For instance, mone reccives the strange pronunciation /moni/ by analogy with anemone. Also, the pseudoword shead receives the bizatre, vowelless pronunciation $/ \int_{-\ldots} \mathrm{d} /$ (where ', denotes the null phoneme) when using the D\&N model and the TWB databasc. As illustrated in Fig. 2 earlicr, this turns out to be a result of matching the unique but long mapping head $\rightarrow /_{-\ldots} \mathrm{d} /$ as in forehead $\rightarrow /$ for $\ldots . .-\mathrm{d} /$ (arc frequency 1) in conjunction with the very common mapping $s h \rightarrow / \int_{-} /$as in she and shed (arc frequency 174) which swamps the overall score of 175 . The same bizarre pronunciation does not occur with the PROD model. In this case, the path through the 
Table 1: Results for $\mathrm{PbA}$ of pseudowords with both dictionaries. See text for further specification.

\begin{tabular}{|c|c||c|c|c|c|}
\hline \multirow{2}{*}{ Test set } & \multicolumn{1}{|c|}{ Implementation } & \multicolumn{2}{c|}{ Webster's (\%) } & \multicolumn{2}{c|}{ TWB (\%) } \\
\cline { 2 - 6 } & & words & phonemes & words & phonemes \\
\hline \hline \multirow{3}{*}{ D\&N 70 } & D\&N & 77.1 & 94.3 & 70.0 & 92.6 \\
\cline { 2 - 6 } & PROD & 82.9 & 95.9 & 78.6 & 94.9 \\
\cline { 2 - 6 } & MP & 85.7 & 96.6 & 80.0 & 95.3 \\
\hline \multirow{2}{*}{ Sull 136 } & D\&N & 75.0 & 93.6 & 72.1 & 93.1 \\
\cline { 2 - 6 } & PROD & 80.1 & 95.0 & 76.5 & 94.5 \\
\cline { 2 - 6 } & MP & 83.8 & 95.9 & 81.6 & 95.7 \\
\hline
\end{tabular}

$(/ \varepsilon /, 3)$ node has a product score of $12 \times 30=360$ for the pronunciation $/ \int \varepsilon d /$ which considerably exceeds the score of 174 for $/ \int \mathrm{d} /$.

Replacing the arc-sum heuristic of the D\&N model by arc-product as in the PROD model leads to a considerable increase in performance, e.g. from $77.1 \%$ words correct to $82.9 \%$ for the D\&N 70 test set with Webster's database. In turn, the MP model performs better than PROD in all cases.

For the Sull 136 test set, our expectation of poorer performance (because of the larger test set and inconsistency between of dialect between the target pronunciations and the lexical databases) is borne out for Webster's dictionary. For TWB, however, the performance difference between test sets is less consistent.

\subsection{Lexical Words}

The primary ability of a text-to-speech system must be to produce correct pronunciations for lexical words (rather than pseudowords) which just happen to be absent from the system's dictionary. Accordingly, we have tested the PbA implementations by removing each word in turn from its relevant database, and obtaining a pronunciation by analogy with the remainder. In these tests, the transcription standard employed by the compilers of the dictionary becomes its own reference and problems of transcription inconsistencies between input strings and lexical entries are avoided.

Results for the testing of lexical words are shown in Table 2. Again there are consistent performance differences with the 'standard' D\&N model worst and the mapping probability (MP) model best. All models perform better with the TWB database than with Webster's, probably simply becausc of its smaller size.

For some lexical words, no pronunciation at all was produced because there was no complete path from Start to End in the lattice. This occurred for 92 of the TWB words and 117 of the Webster's words irrespective of the scoring model. This is a scrious shortcoming: a PbA system should always produce a bestattempt pronunciation, even if it cannot produce the correct one. Sometimes, this failure is a consequence of the form of pronunciation lattice in which nodes are used to represent the 'end-points' of mappings. One of the inputs for which no pronunciation was found is anecdote, whose (partial) lattice is shown in Fig. 3. There is in fact no arc in the complete lattice between nodes $(/ \mathrm{k} /, 4)$ and $(/ \mathrm{d} /, 5)$ because there is no $c d \rightarrow / \mathrm{kd} /$ mapping anywhere in either dictionary. Nor is there an ecd or cdo trigram - with or without the right endpoint phonemes - which could possibly bridge the gap. This problem is entirely avoided with the Sullivan and Damper style of lattice, because the shortest-length arc corresponds to a single-symbol mapping rather than to a bigram (which may be unique). Thus, there will always be a 'default' single-symbol mapping corresponding to the commonest pronunciation of the letter. This is not to say that Sullivan and Damper's system will necessarily produce the correct output here: it almost certainly will not because of the rarity of the $c \rightarrow / \mathrm{k} /$ mapping in the $-d$ context.

Another input which fails to produce a pronunciation is aurdvark. The problem here is not that there is no a bigram in the dictionary (which is found in words such as bazaar), but that it only appears towards the end of other words. Dedina and Nusbaum's strategy of performing substring matching only over a restricted range (the number of matching comparisons is equal to the difference in length between the input string and lexical entry) is at the root of this problem.

\section{Conclusions and Discussion}

We find that Dedina and Nusbaum's reported error rate of $9 \%$ cannot be reproduced: our figure is about two or three times that. Because of the shortcomings which emerge in this work, we believe the problem lies with PRONOUNCE rather than our reimplementation. Overall, our results are in much closer agreement with Sullivan and Damper's word error rates of almost $30 \%$ on a similar test set.

This work suggests several useful ways in which the performance of $\mathrm{PbA}$ systems might be improved. Our best results are obtained with a scoring method based on a priori mapping probabilities. According to Sul- 
Table 2: Results for PbA of dictionary words.

\begin{tabular}{|c||c|c|c|c|}
\hline \multicolumn{1}{|c||}{ Implementation } & \multicolumn{2}{c|}{ Webster's (\%) } & \multicolumn{2}{c|}{ TWB (\%) } \\
\cline { 2 - 5 } & words & phonemes & words & phonemes \\
\hline \hline D\&N & 57.8 & 90.4 & 65.6 & 93.1 \\
\hline PROD & 58.5 & 90.7 & 66.1 & 93.2 \\
\hline MP & 60.7 & 91.2 & 67.9 & 93.5 \\
\hline
\end{tabular}
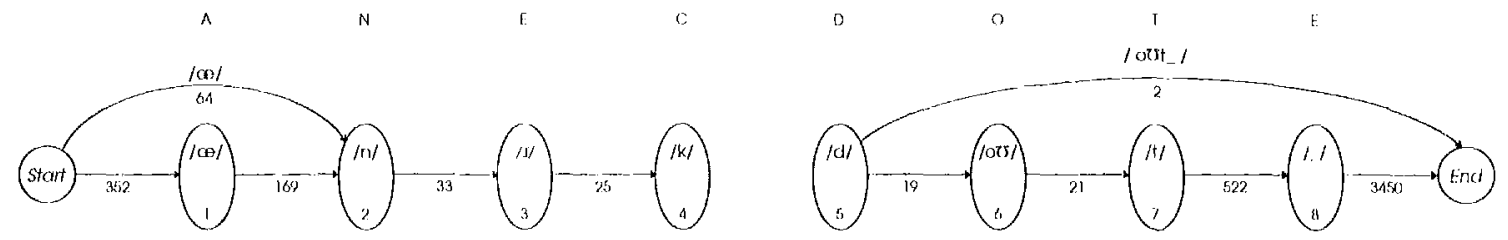

Figure 3: Simplified pronunciation lattice for the lexical word anecdote which fails to produce any pronunciation.

livan and Damper (1993), a posteriori mapping probabilities may do even better. Also, the type of pronunciation lattice used by Sullivan and Damper, in which nodes correspond to the junctures between symbols, is likely to be superior. The impact of different alignment strategies should repay study. Finally, we intend to assess the impact of incorporating information about word frequency in the analogy process.

\section{Acknowledgement}

This work was funded by the UK Ficonomic and Social Research Council via research grant R000235487: "Speech Synthesis by Analogy".

\section{References}

Baron, J. (1977). Mechanisms for pronouncing printed words: use and accuisition. In Busic l'rocesses in Reading: Perception and Comprehension (1). LaBerge and S. Samucls, eds.), pp. 175-216. Lawrence Litlyaum, Hillsdale, NJ.

13rooks, L. (1977). Non-analytic correspondences and pattern in word pronunciation. In Attention and Performance VII (J. Rencuin, cl.), pp. 163-177. I awrence lirlbaum, Hillsdalc, NJ.

Brown, P. and Besner, D. (1987). 'The assembly of phonology in oral reading: a new model. In Attention and Performance XII: the Psychology of Reading (M. Coltheart, ed.), [p. 471-489. Lawrence Erlbaum, London.

Colthcart, M. (1978). Lexical aceess in simple reading tasks. In Strategies of Information Processing (G. Underwood, ed.), pp. 151-216. Academic, London.

Dedina, M.J. and Nusbaum, II.C. (1991). PRONOUNCE: a program for pronunciation by analogy. Compuier Specch and language, 5, 55-64.

Glushko, R.J. (1979). The organization and activation of of thographic knowledge in reading aloud. Journal of Expert mental Psychology: Human Perception and Performance, 5, $674-691$.
Glushko, R.J. (1981). Principles for pronouncing print: the psychology of phonography, In Interactive Processes in

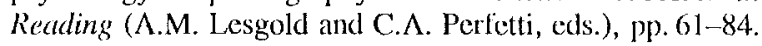
Lawrence Erlbaum, Hillsdale, NJ.

Humphreys, G.W. and Evett, I ..J. (1985), Are there independent lexical and nonlexical routes in word processing? An evaluation of the dual-route theory of reading. Behavioral and Brain S'iences, 8, 689-740.

Lawrence, S.G.C. and Kaye, G. (1986). Alignment of phonemes with their corresponding orthography. Computer Sipech and Language, 1, 153-165.

Levenshtein, V.I. (1966). Binary codes capable of correcting deletions, insertions and reversals. Cybernetics and Control Theory, 10, 707-710.

McCulloch, N., Bedworth, M. and Bridle, J.S. (1987). NETspeak - a re-implementation of NETtalk. Computer Speech and Language, 2, 289-301.

Scjnowski, 'T.J. and Rosenberg, C.R. (1987). Parallel networks that learn to pronounce English text. Complex Systems, 1, 145-152.

Sullivan, K.P.H. (1992). Synthesis-by-Analogy: a Psychologically Motivated Approach to Text-to-Speech Conversion, PhD Thesis, Department of Electronies and Computer Science, University of Southampton, UK.

Sullivan, K.P.H. and Damper, R.I. (1990). A psychologically governed approach to novel-word pronunciation within a text-10-speceh system. Proceedings of IEEE International Conference on Acoustics, Speech and Signal Processing (ICASSP' '90), Vol. 1, Albuquerque, NM, pp. 341-344.

Sullivan, K.P.IY, and Damper, R.I. (1992). Novel-word pronunciation within a text-to-speech system. In Talking $\mathrm{Ma}$ chines: Theories, Models and Applications (G. Bailly and C. Benoît, eds.), pp. 183-195. Lilsevier (Notth-L Lolland), Amsterdam.

Sullivan, K.P.H. and Damper, R.I. (1993). Novel-word prounuciation: a cioss-language study. Speech Communication, $13,441 \cdots 452$

'I'horndike, L.I. and Lorge, I. (1944). The Teachers' Word book of 30,000 Words. 'Teachers' College, Columbia University, NY. 